\title{
Infiltración asintomática de la próstata por leucemia linfática crónica
}

\author{
Teresa Pastor Navarro, Jorge Planelles Gómez, Manel Beamud Cortés, Encarna Martí Ibor, \\ Manuel Gil Salom, Jose Manuel Osca García
}

Servicio de Urología. Hospital Universitario Doctor Peset. Valencia, España

\begin{abstract}
Resumen
Objetivo: Presentar un caso de infiltración leucémica de la próstata como hallazgo incidental en una prueba de imagen. Métodos: Describimos el caso de un varón de 60 años en seguimiento por una leucemia linfática crónica, al que se realiza un TAC de rutina apreciando adenopatías múltiples y un nódulo hipodenso en el lóbulo prostático izquierdo. El paciente es remitido a nuestro servicio para la realización de ecografía transrectal y biopsias prostáticas. No refiere clínica urinaria.

Resultados: Al tacto rectal la próstata está mal delimitada y con aumento difuso de consistencia. La ecografía transrectal muestra una glándula mal delimitada, con parénquima heterogéneo y varias áreas hiperecoicas. El informe anatomopatológico describe la infiltración difusa de la glándula por una leucemia linfática crónica.

Conclusiones: Aunque la extensión de tumores hematológicos a la próstata ha sido descrita previamente, no existe en nuestro conocimiento ningún caso publicado de hallazgo incidental en una prueba de imagen de infiltración prostática por leucemia en un paciente asintomático.
\end{abstract}

Palabras clave: Infiltración prostática. Leucemia. Nódulo prostático. Asintomático.

\section{Assymptomatic prostatic infiltration by chronic lymphocytic leukemia}

\section{Abstract}

Objective: Report of a case of leukemic infiltration of the prostate as an incidental CT finding.

Methods: We describe a case of a 60-year-old male suffering from chronic lymphocytic leukemia. A routine-performed CT showed multiple lymphadenopathies and an hypodense area in the left prostatic lobe. The patient was sent to our department to carry out a transrectal ultrasound and prostatic biopsy. He didn't relate any urinary symptoms.

Results: By the prostate rectal examination the gland felt hard and with no well defined limits. The transrectal ultrasound showed heterogene parenchyma and several hyperechoic areas by a bad circumscribed prostate. The pathological analysis reported a diffuse infiltration of the gland by chronic lymphocytic leukemia cells.

Conclusions: Although the relapse of hematological tumors to the prostate has been described previously, there is no published case to our knowledge of a leukemic prostate infiltration in an assymptomatic patient as an incidental finding by an imaging procedure.

Keywords: Prostate infiltration. Leukemia. Prostate node. Assymptomatic.

$\mathrm{L}^{2}$ a extensión de tumores hematológicos al aparato urinario no es infrecuente, sin embargo la infiltración prostática es inhabitual. En la mayoría de los casos descritos en la literatura se trata de pacientes que han presentado sintomatología miccional y mediante biopsia prostática se ha detectado la infiltración por el tumor, ya fuera éste conocido previamente o no. Presentamos un caso de un paciente diagnosticado de Leucemia Linfática Crónica en estadio A/0 y seguimiento por el Servicio de Hematología de nuestro centro, sin clínica miccional, al cual se detectó incidentalmente en un TAC de control una masa prostática que tras biopsia fue diagnosticada de infiltración por la leucemia. Es el primer caso publicado que se presenta sin sintomatología urinaria y es descubierto de forma incidental en una prueba de imagen.

\section{CASO CLÍnICo}

Varón de 60 años con antecedentes de hipertensión arterial, valvulopatía aórtica y mitral, hematoma parietal izquierdo, herniorrafia inguinal izquierda y umbilical, diagnosticado hace 3 años de Leucemia Linfática Crónica estadio A/O y en segui- 
miento por el servicio de Hematología de nuestro hospital, sin tratamiento. En un TAC toracoabdominopélvico de control se aprecian adenopatías a nivel cervical, axilar, mediastínico, retroperitoneal e inguinal, así como una imagen hipodensa de $3 \mathrm{~cm}$ el lóbulo prostático izquierdo, y con mal plano de separación con el músculo elevador del ano izquierdo (Figs. 1 y 2), por lo que ante la sospecha de neoplasia prostática se solicita analítica de PSA y se remite a nuestro servicio para valoración y realización de ecografía transrectal y biopsias prostáticas. El paciente no presenta clínica urológica.

En el tacto rectal se aprecia una próstata mal delimitada en ambas bases, con aumento de consistencia de forma difusa en ambos lóbulos. El PSA es de $0,9 \mathrm{ng} / \mathrm{ml}$. La ecografía transrectal muestra una próstata de 28,5 x 43,4 x 50,8 mm y 32,9 ml, con parénquima heterogéneo y varias áreas hiperecoicas en ambas bases. El ángulo vesiculoprostático derecho aparece borrado. Se toman biopsias prostáticas por sextantes.

El informe anatomopatológico describe un parénquima prostático con denso infiltrado celular constituído por linfocitos de pequeño tamaño sugestivo de infiltración por leucemia linfática crónica (Figs. 3 y 4).

\section{DISCUSIÓN}

La LLC es una neoplasia hematológica monoclonal, de linfocitos $\mathrm{B}$ generalmente, y es el tipo más frecuente de leucemia en ancianos. Existen varias formas de estadiaje, de las cuales las 2 más utilizadas son la clasificación de Rai (estadios 0 a IV) y la de Binet (estadios A a C). En el estadio 0/A se incluyen las neoplasias asintomáticas que sólo producen linfocitosis y afectan a menos de 3 áreas linfoides, y que requieren actitud expectante sin tratamiento ${ }^{1}$.

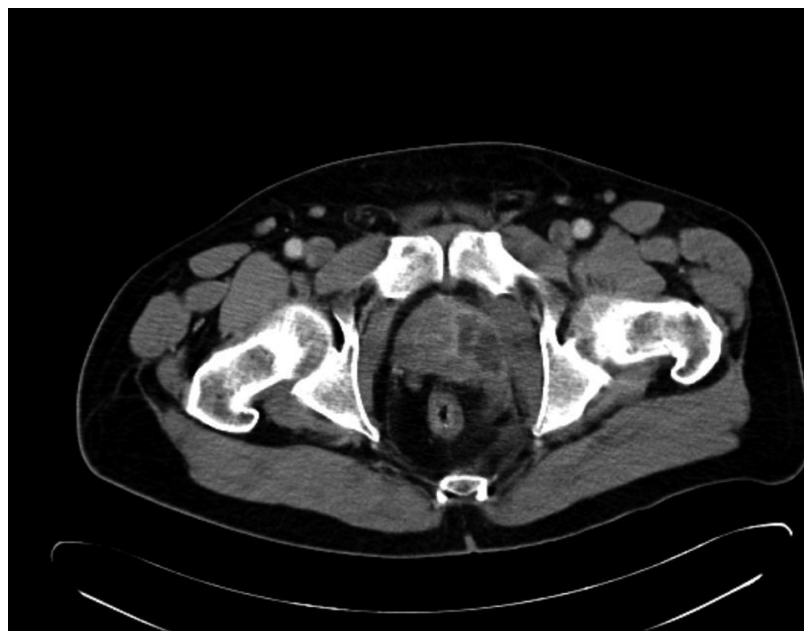

FIGURAS 1 y 2. TAC: Masa prostática en el lóbulo izquierdo con mal plano de separación con el músculo elevador del ano izquierdo.
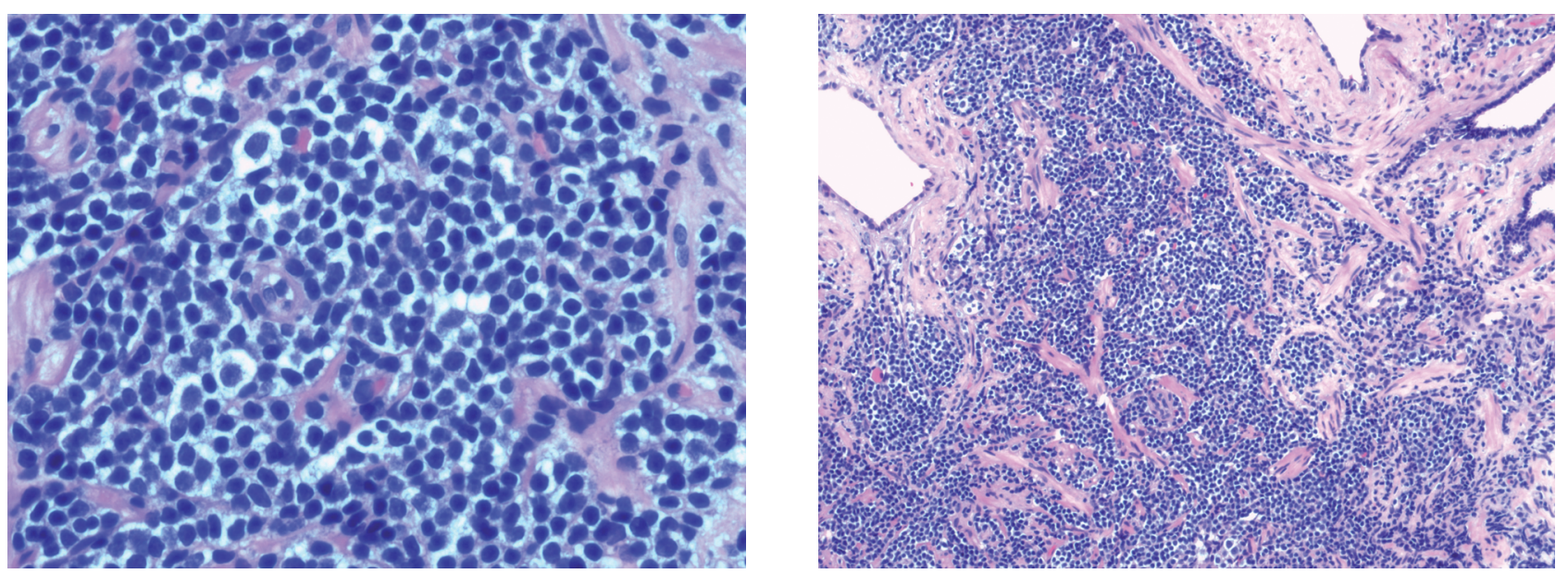

FIGURAS 3 y 4. Biopsias prostáticas: infiltrado celular constituído por linfocitos de pequeño tamaño. 
La existencia de múltiples adenopatías y la extensión del tumor a la próstata suponen un cambio de estadio, con un consiguiente empeoramiento del pronóstico y que justifica el inicio de tratamiento quimioterápico ${ }^{1}$. La LLC es la causa más frecuente de infiltración de la próstata por neoplasias de origen hematológico ${ }^{2}$.

En la literatura existen pocos casos publicados de afectación prostática por neoplasias hematológicas. El primer caso indexado es el descrito por Johnson y Gundersen en 1953 en The Journal of Urology ${ }^{3}$. Los casos más frecuentemente relatados son pacientes que presentan clínica miccional ${ }^{4-6}$, y especialmente retención urinaria ${ }^{7-12}$, o alteración de las cifras de $\mathrm{PSA}^{13}$, a raiz de lo cual se diagnostica la infiltración leucémica de la glándula, ya fuera la neoplasia previamente conocida o no. Algunos de estos pacientes además están afectos sincrónicamente de carcinoma prostático $7,9,14$. No obstante las citas anteriores, cabe destacar que el hallazgo radiológico incidental de una masa leucémica en próstata sin clínica acompañante nunca ha sido descrito en la literatura.

\section{REFERENCIAS}

1. Freedman AS, Nadler LM. Neoplasias de las células linfoides. In Fauci AS, Braunwald E, Isselbacher KJ, Wilson JD, Martin JB, Kasper DL et al. Harrison Principios de medicina interna $14^{\mathrm{a}}$ edición. Madrid, McGraw-Hill, 1998, pp 793-812.

2. Epstein J. Anatomía patológica de la neoplasia prostática. In Walsh PC, Retik AB, Vaughan ED, Wein AJ. Campbell Urología. Madrid, Panamericana. 2005, pp 3313-3326.

3. Johnson MA, Gundersen AH. Infiltration of the prostate gland by chronic lymphatic leukemia. J Urol. 1953;69(5):681-685.

4. Maruyama T, Yamamoto S, Nojima M, Morita N, Tanizawa T, Shima SH. Extramedullary relapse of acute lymphoblastic leukemia in childhood to the prostate. Int J Urol. 2007;14(5): 447 449 .
5. Gunlusoy B, Cicekl S, Selek E, Sayhan S, Minareci S, Arslan M. A case report: Leukaemic infiltration and hyperplasia of the prostate. Int Urol Nephrol. 2004;36(1):55-56.

6. Eddes EH, Fibbe WE, Loyson SA, Zwartendijk J. Urinary symptoms due to leukemic infiltration of the prostate. A case report. Ann Hematol. 1993;66(6):323-324.

7. Ballario R, Beltrami P, Cavalleri S, Ruggera L, Zorzi MG, Artibani W. An unusual pathological finding of chronic lymphocitic leukemia and adenocarcinoma of the prostate after transurethral resection for complete urinary retention: case report. BMC Cancer. 2004;4:95.

8. Yavuz S, Paydas S, Disel U, Zorludemir S, Erdogan S. Prostatic hypretrophy and prostatic infiltration in small lymphocytic lymphoma. Leuk Lymphoma. 2004;45(1):201-202.

9. Ather MH, Memon A. Acute urinary retention: a primary manifestation of chronic lymphocytic leukemia and organ confined prostate cancer. J Pak Med Assoc. 1999;49(5):124-125.

10. Hope-Gill B, Goepel JR, Collin RC. Obstructive uropathy associated with myelomonocytic infiltration of the prostate. J Clin Pathol. 1998;51(4):340-342.

11. Benekli M, Büyükasik Y, Haznedaroglu IC, Savas MC, Ozcebe OI. Chronic lymphocytic leukemia presenting as acute urinary retention due to leukemic infiltration of the prostate. Ann Hematol. 1996;73(3):143-144.

12. Singh G, Murray K. Lymphocytic infiltration of the prostate presenting as retention. Urol Int. 1994;53(4):225-226.

13. Pérez Arbej JA, López Carreira M, Arnáiz Esteban F, Martínez Pérez E, Nogueras Gimeno MA, Espuela Orgaz R, et al. Diagnosis of leukemic infiltration of the prostate prompted by PSA elevation. Actas Urol Esp. 1996;20(10):892-894.

14. Terris MK, Freiha FS. Transrectal ultrasound appearance of hematolymphoid malignancies involving the prostate. Urology. 1998;51(2):339-341.

Correspondencia autor: Dra. Teresa Pastor Navarro Servicio de Urología

Hospital Universitario Doctor Peset

Gaspar Aguilar 90. 46017 - Valencia

Tel. y Fax: 961622403

E-mail autor: tpastorn@yahoo.es

Información artículo: Nota clínica

Trabajo recibido: noviembre 2008

Trabajo aceptado: diciembre 2008 